\title{
Design and Performance of SEFDM Signals with Power Allocation
}

\author{
Hedaia Ghannam and Izzat Darwazeh \\ Department of Electrical and Electronics Engineering \\ University College London, London, United Kingdom \\ E-Mail: hedaia.ghannam.15@ucl.ac.uk, i.darwazeh@ucl.ac.uk
}

\begin{abstract}
This work presents preliminary investigations into the use of power allocation for the multi-carrier nonorthogonal spectrally efficient frequency division multiplexing (SEFDM) signalling format. SEFDM is utilized to improve the spectral efficiency compared to conventional orthogonal frequency division multiplexing (OFDM), by violating the orthogonality condition and getting the subcarriers closer to each other. In this paper, subcarriers within the same SEFDM symbol are allocated different power levels. Results show that such power allocation is beneficial to SEFDM from several perspectives: $i$ ) Overall system stability enhancement; ii) a drastic complexity reduction in SEFDM detector; iii) peak to average power ratio (PAPR) performance improvement.
\end{abstract}

\section{INTRODUCTION}

One of the main challenges of future communication systems is to improve the spectral efficiency. Many techniques were proposed for the purpose of improving spectral efficiency relative to that of the well known orthogonal frequency division multiplexing (OFDM). Spectrally efficient frequency division multiplixing (SEFDM) was originally proposed in 2003 [1] and the idea behind it is to pack more subcarriers, relative to OFDM, in a given bandwidth to improve capacity [2]. Orthogonality violation in SEFDM results in inter-carrier interference (ICI) and creates an environment with substantial distortion that will be severely damaging if left uncompensated [3].

A key question is: Why hiring SEFDM if OFDM signals spectral efficiency can be improved by simply increasing the cardinality order of the symbols? In the literature, it was shown that the use of SEFDM signals enhances the error performance, when compared to OFDM of the same throughput [4] [5]. Furthermore, SEFDM peak to average power (PAPR) characteristics are better than OFDM [6] [7]. The efficacy of SEFDM was proven in experimental test beds for wireless [4], optical/mm-wave [8], VLC system [9], very high speed optical [10], [11] and the downlink for Internet of Things (IoT) networks [12].

The use of power allocation in OFDM has been excessively studied and shown to have advantages in improving capacity and robustness to interference caused by multipath channels ( see [13] and the references therein). More recently, the non-orthogonal multiple access (NOMA) technique has attracted substantial attention, as it increases the overall system throughput [14]. NOMA allows multiple users to share time and frequency resources via power domain or code domain multiplexing [14]. Inspired by the power domain multiplexing of NOMA, this work, and for the first time, introduces a similar power allocation method to SEFDM, where different subcarriers within the same SEFDM symbol are allocated different power levels. Such power allocation is beneficial to SEFDM from various aspects: First, the essential limitation of SEFDM is in its correlation matrix (similar to the correlation matrix in massive multi-input multi-output (MIMO) systems) resulting from the interference between subcarriers. The condition number of this matrix is substantially high, especially with high compression level and/or relatively high number of subcarriers, thus, the system becomes ill-conditioned. Power allocation is capable of solving this problem and enhances the system stability by reducing the condition number of the correlation matrix and making it independent of the number of subcarriers [15]. The second advantage is the reduction of the detector complexity. Finally, power allocation results in PAPR reduction compared to conventional SEFDM and OFDM signals.

In this work, we investigate the three aforementioned advantages of power allocation, where two power levels are allocated for different sets of subcarriers. The detection of the SEFDM signal with power allocation is processed through two stages. First, the information transmitted on the subcarriers of high-power level is estimated and the interference coming from these subcarriers to the rest of the subcarriers is calculated and then cancelled. Second, The low-power level subcarriers are detected using a unity level fixed sphere decoder (FSD) [16].

The outline of this paper is as follows; we start with introduction of SEFDM signals with power allocation in section II. The advantages of power allocation utilization 


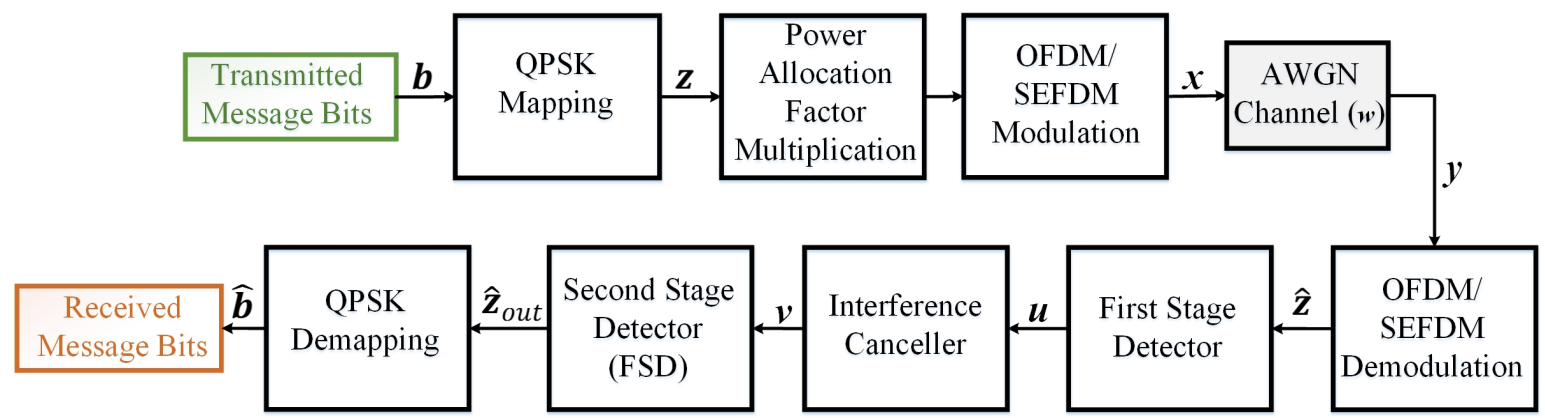

Fig. 1: A high level descriptive transceiver design of SEFDM signals with power allocation.

are investigated in section III. Finally, conclusions are drawn in section IV.

Notation : Matrices are denoted by bold uppercase letters, bold lowercase letters represent column vectors. $(.)^{\mathrm{H}}$ is the Hermitian operator, $\lceil$.$\rceil is the ceiling function,$ [.] is the slice function and $\|$.$\| is the Euclidean norm.$

\section{Power Allocation Method for SEFDM}

SEFDM is a multi-carrier modulation technique, where symbols are generated similarly to OFDM. The equation below represents the discrete SEFDMmodulated symbol vector, $\mathbf{x} \in \mathbb{C}^{Q \times 1}$, at the transmitter side [8]

$$
\mathbf{x}=\frac{1}{\sqrt{Q}} \sum_{n=0}^{N-1} z_{n} \exp \left(j 2 \pi \alpha \frac{q n}{Q}\right), 0 \leq q \leq Q-1
$$

where $\mathbf{z} \triangleq\left\{z_{0}, z_{1}, . .,, z_{N-1}\right\}$ is the complex baseband symbols to be modulated by $N$ subcarriers of the SEFDM symbol, $Q \geq N$ is the total number of samples per SEFDM symbol and $1 / \sqrt{Q}$ is employed for normalization purposes. $\alpha \in(0,1]$ is the compression factor, hence an SEFDM system saves $(1-\alpha) \times 100 \%$ of bandwidth relative to an equivalent OFDM system, where $\alpha=1$.

If the SEFDM signal in (1) were to be transmitted in additive white Gaussian noise (AWGN), with zero mean and variance $\sigma_{n}^{2}=\mathrm{N}_{0} / 2$, where $\mathrm{N}_{0}$ is the noise power spectral density, the SEFDM-received signal $\mathbf{y} \in \mathbb{C}^{Q \times 1}$ can be presented by

$$
\mathbf{y}=\mathbf{x}+\mathbf{w}
$$

where $\mathbf{w} \in \mathbb{C}^{Q \times 1}$ consists of the AWGN samples. A high level descriptive transceiver design of SEFDM signals with power allocation transmission is shown in Fig. 1. At the receiver, the demodulated symbol $\hat{\mathbf{z}} \in \mathbb{C}^{N \times 1}$, affected by the channel and contaminated by ICI, is given by [8]

$$
\begin{aligned}
\hat{\mathbf{z}} & =\frac{1}{\sqrt{Q}} \sum_{q=0}^{Q-1} y_{q} \exp \left(-j 2 \pi \alpha \frac{q n}{Q}\right), 0 \leq n \leq N-1, \\
& =\mathbf{\Lambda} \mathbf{z}+\mathbf{w}
\end{aligned}
$$

where $\boldsymbol{\Lambda} \in \mathbb{C}^{N \times N}$ is the correlation matrix with a diagonal of ones and non-diagonal elements $\left(\Lambda_{m, n}\right)$ representing the interference from subcarrier $n$ on subcarrier $m$ and given by [17]

$$
\begin{aligned}
& \Lambda(m, n)=\exp (j \pi \alpha(m-n)) \times \\
& \exp \left(\frac{-j \pi \alpha(m-n)}{Q}\right)\left[\frac{\operatorname{sinc}(\alpha(m-n))}{\operatorname{sinc}\left(\frac{\alpha(m-n)}{Q}\right)}\right] .
\end{aligned}
$$

For a detailed mathematical treatment of ICI in SEFDM, readers are referred to [17].

The proposed power allocation method is characterised by transmitting subcarriers at different power levels. This is based on the fact that within the SEFDM symbol, there are subcarriers that may be mutually orthogonal. For $\alpha=b / c$ where $b$ and $c$ are integers, $b<c$ and for $c<N$, there are $\lceil N / c\rceil$ mutually orthogonal subcarriers $\left(\left|\Lambda_{m, n}\right|=0\right)$ for $\alpha(m-n) \in \mathbb{Z}$. Thus, a subset of mutually orthogonal subcarriers will be transmitted at higher power level $\left(P_{H}\right)$ as the subcarriers in this subset will not interfere with each others. The rest of the subcarriers are allocated with the lower power level $\left(P_{L}\right)$. In order to have a fair comparison with SEFDM signals without power allocation, it is

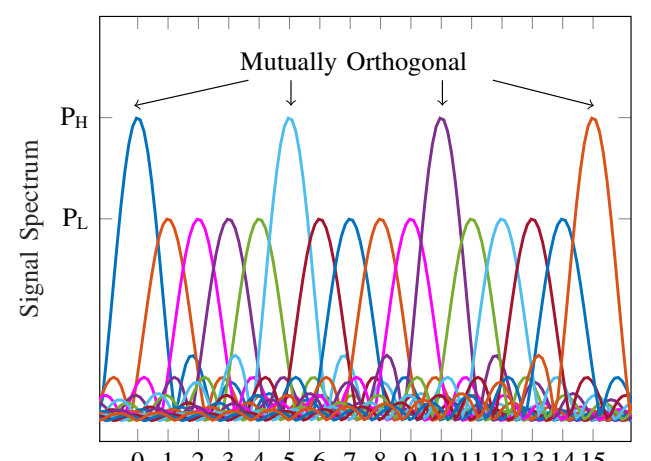

subcarrier index (n)
Fig. 2: SEFDM signal spectrum with power allocation for $N=16, \alpha=4 / 5, b=4$ and $c=5$. 


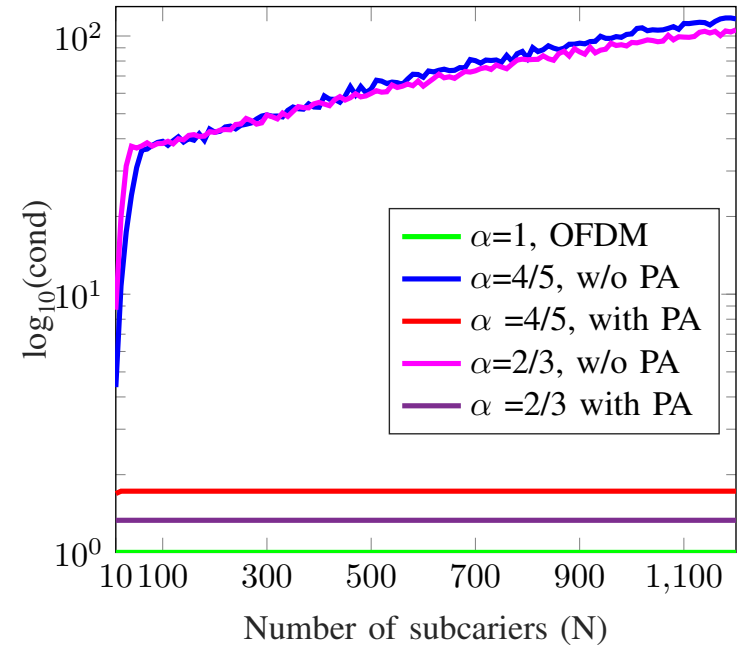

Fig. 3: Condition number versus $N$ for different values of $\alpha$, with/without power allocation (PA).

important to maintain the same total transmitted power (i.e. $P=\mathbf{x}^{\mathrm{H}} \mathbf{x}$ ). Thus, the increase in power level in some subcarriers results in a reduction of the power level for the rest, such as the total power $P$ remains unchanged;

$$
P=P_{H}\lceil N / c\rceil+P_{L}(N-\lceil N / c\rceil) .
$$

The parameter $\rho$ is defined as the ratio between the upper and lower power levels $P_{H} / P_{L}$ and $\rho$ optimum value is a function of the system parameters $(N$ and $\alpha)$. From Fig. 1, prior to SEFDM modulation, the multiplication of the complex symbol $z_{n}$ by one of the two weighting factors will result in the desired power allocation.

A visual representation of power allocation is given in Fig. 2, where the spectrum of an SEFDM symbol with $N=16, \alpha=4 / 5$ is shown. In this case, $b=4$ and $c=5$, which results in 4 subcarriers of power $P_{H}$ and the rest of power $P_{L}$.

At the receiver, the mutually orthogonal subcarriers are detected first, by taking a hard decision on these high-power level subcarriers while the rest are set to zero as shown below

$$
u_{n}= \begin{cases}\left\lfloor\hat{z}_{n}\right\rceil, & n \bmod c=0 \\ 0, & \text { otherwise }\end{cases}
$$

where mod is the modulo operation. Thereafter, $\mathbf{u} \in$ $\mathbb{C}^{N \times 1}$ is used to calculate and cancel the interference from the subcarriers with high-power on the rest of the low-power subcarriers, as shown in (7). This interference cencellation method is similar to the one of [5], which was applied successfully with iterative interference canceller and turbo equaliser in an SEFDM satellite scenario. The resultant vector $\mathbf{v} \in \mathbb{C}^{N \times 1}$ is the input to a second stage suboptimal low complex detector.

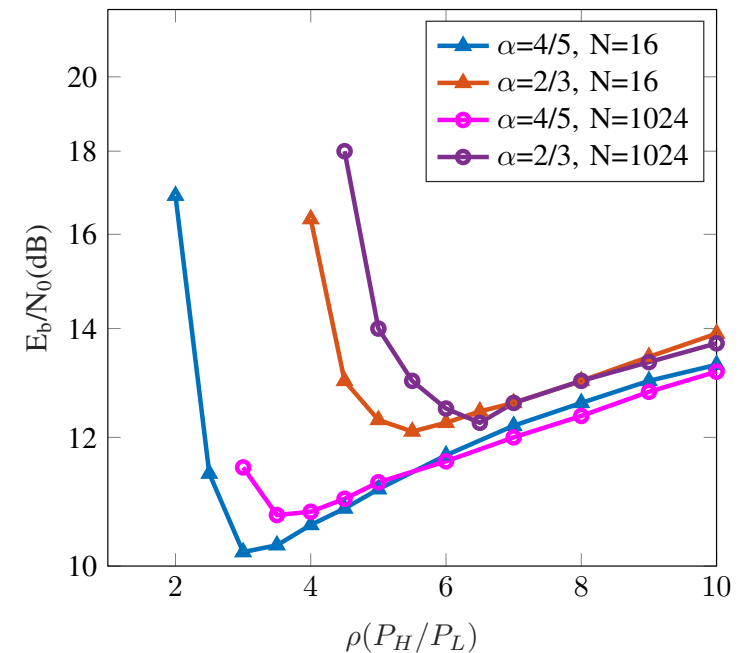

Fig. 4: $E_{b} / N_{0}$ required to achieve a $\mathrm{BER}=10^{-3}$ versus $\rho$, for different values of $N$ and $\alpha$.

$$
\mathbf{v}=\hat{\mathbf{z}}-\boldsymbol{\Lambda} \mathbf{u} .
$$

As a consequence of first stage interference cancellation, the correlation matrix $\Lambda$ is updated to $\Lambda$, where the elements describing the interference already cancelled in the first stage are set to zero, and this is given by

$$
\Lambda_{m, n}= \begin{cases}0, & n \bmod c=0 \\ \Lambda_{m, n}, & \text { otherwise }\end{cases}
$$

This adjustment to the correlation matrix results in orders of magnitude reduction in its condition number as will be shown in the next section, where the advantages of assigning the power allocation method to SEFDM are investigated.

\section{Power Allocation Advantages}

\section{A. Condition Number Reduction}

When the condition number of the correlation matrix $(\boldsymbol{\Lambda})$ is very large, then the matrix becomes illconditioned and almost singular. The computation of the inverse of an ill-conditioned matrix for a linear detector will be prone to large numerical errors in such case. In SEFDM, the condition number of $\boldsymbol{\Lambda}$ increases rapidly with increasing the compression level and/or the number of subcarriers [15].

Fig. 3 demonstrates the logarithmic value of the condition number $(\log ($ cond $))$ versus $N$ for different values of $\alpha$. The figure shows that the resultant correlation matrix $\Lambda$ has a drastic reduction in its condition number compared to conventional SEFDM and it is independent of $N$, similar to the OFDM case.

The reward of this correlation matrix adjustment is highly significant for sub-optimal and low complex 


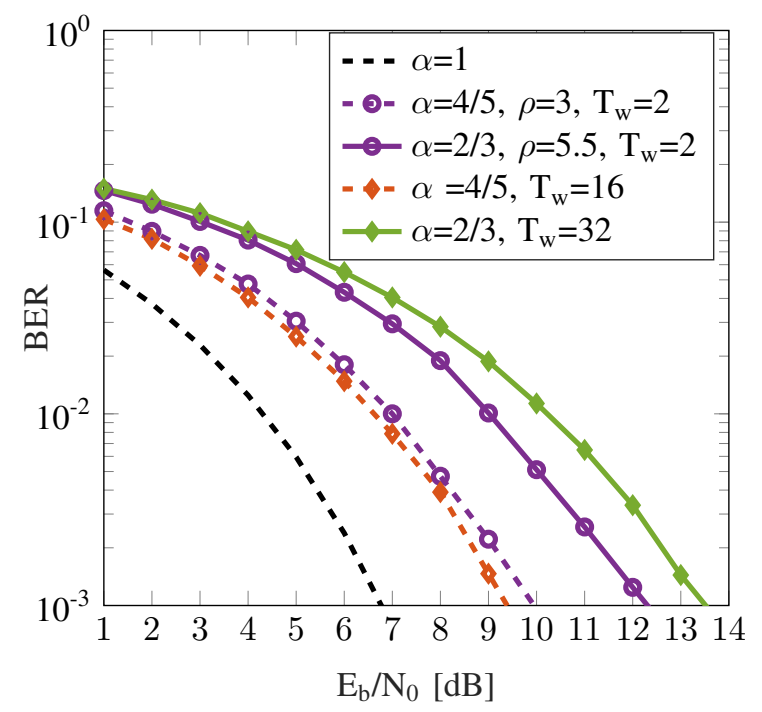

(a) $N=16$

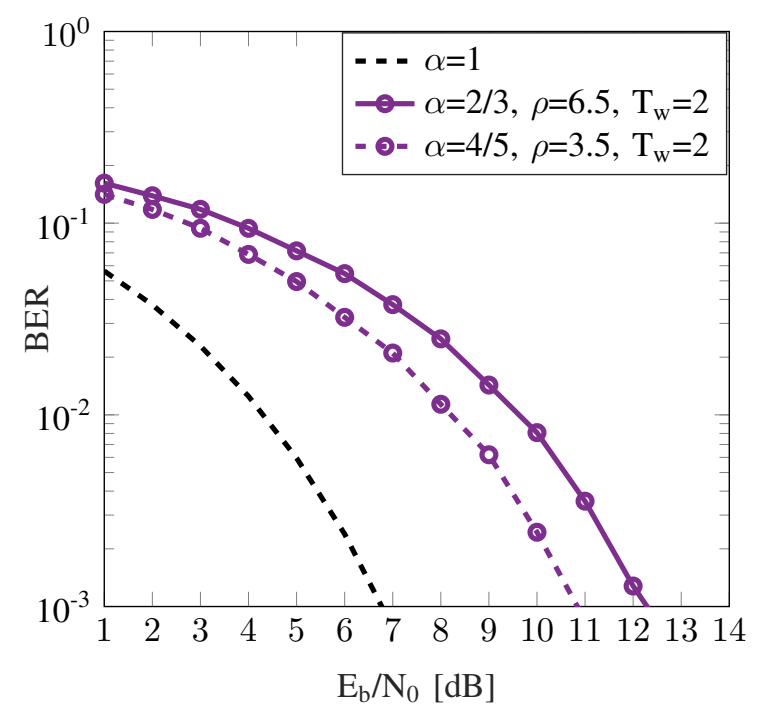

(b) $N=1024$

Fig. 5: A comparison of SEFDM signals BER performance with and without power allocation for $M=4$, $\alpha=4 / 5,2 / 3$ and (a) $N=16$, (b) $N=1024$.

detectors that may be implemented in practice. For instance the FSD, which will be used in the next section as a second stage detector, requires an inversion of the correlation matrix for an initial zero forcing (ZF) estimate and it is crucial in determining the performance of the system. If the initial estimate is compromised by contributions of the ill-conditioned system then the deviations from the received statistics point will propagate to the final solution.

\section{B. Complexity Reduction}

The FSD algorithm fixes the complexity of SD by restricting the search within a limited subspace of the problem. At every level $(k)$, a fixed number of nodes, or in other words tree width $\left(T_{w}=2^{k}\right)$, are examined. The FSD estimate $\left(\hat{\mathbf{z}}_{\text {out }}\right)$ is obtained from the minimisation problem stated in [18]

$$
\hat{\mathbf{z}}_{\text {out }}=\arg \min _{\tilde{\mathbf{z}} \subset \mathcal{H}, \tilde{z} \in M}\|\mathbf{L}(\mathbf{p}-\tilde{\mathbf{z}})\|^{2} \leq \breve{g},
$$

where $\tilde{\mathbf{z}}$ is a candidate SEFDM symbol within the search subspace $\mathcal{H}, M$ is the constellation cardinality, $\mathbf{L}$ is an upper triangular matrix defined by the Cholesky decomposition as $\hat{\Lambda}^{\mathrm{H}} \hat{\Lambda}=\mathbf{L}^{\mathrm{H}} \mathbf{L}$ and $\mathbf{p}$ is [18]

$$
\mathbf{p}=\left(\boldsymbol{\Lambda}^{\mathrm{H}} \hat{\Lambda}\right)^{-1} \hat{\Lambda} \mathbf{v}
$$

$\breve{g}$ is the radius of the search sphere, which corresponds to the distance from the ZF estimate $\hat{\mathbf{z}}_{\mathrm{ZF}}=\left\lfloor\boldsymbol{\Lambda}^{-1} \mathbf{v}\right\rceil$ and is given by [18]

$$
\breve{g}=\left\|\mathbf{v}-\hat{\Lambda} \hat{\mathbf{z}}_{\mathrm{ZF}}\right\|^{2} .
$$

From the above, it is noticed that the first stage detector affects both the $\mathrm{ZF}$ estimate $\hat{\mathbf{z}}_{\mathrm{ZF}}$ and the correlation matrix for FSD detection.

As mentioned earlier, the power ratio $\rho$ depends on $N$ and $\alpha$. The optimum $\rho$ is the value that minimizes the required energy per bit $E_{b}$ over $N_{0}\left(E_{b} / N_{0}\right)$ value for $a$ given error performance. Numerical simulations are used to find out the optimum ratio. Fig. 4 demonstrates the $E_{b} / N_{0}$ value required to achieve a bit error rate (BER) of $10^{-3}$, using the two stages detector of Fig. 1, with $T_{w}=2$ for the FSD. It can be noticed that the optimum value of $\rho$ shifts towards higher values for higher $N$ and/or lower $\alpha$. Furthermore, the starting $\rho$ value varies as well, because it has been found that the BER curve hits an error floor at values higher than $10^{-3}$, as the error caused by ICI is the dominant one in this region.

Consequently, the optimum $\rho$ values are then used to evaluate and compare the BER performance of the new method to conventional SEFDM. Fig. 5 demonstrates the BER results versus $\mathrm{E}_{\mathrm{b}} / \mathrm{N}_{0}$ for $M=4, \alpha=4 / 5,2 / 3$, for small and large number of subcarriers. The FSD is of unity level for power allocation, however, the FSD detector becomes more complex and of higher level order for conventional SEFDM. From the results, it can be noticed that the tree width of conventional FSD (diamond marker in Fig. 5(a)) needs to be increased substantially to achieve BER performance comparable to the new method with power allocation and unity level FSD. For instance, when $N=16$, the tree width increases to 16 and 32 for $\alpha=4 / 5$ and $\alpha=2 / 3$, respectively, compared to $T_{w}=2$. For $N=1024$ only the results with power allocation are shown, as for 
TABLE I: Computational complexity (in terms of number of real operations).

\begin{tabular}{|l|l|}
\hline & Number of operations \\
\hline First stage detector multiplications & $4 N^{2}$ \\
First stage detector additions & $4 N^{2}$ \\
\hline Second stage (FSD) multiplications & $\sum_{n=1}^{w} 2^{n}[2 n+1]+\sum_{m=w+1}^{2 N} T_{w}[2 m+1]$ \\
Second stage (FSD) additions & $\sum_{n=1}^{w} 2^{n}[2 n-1]+\sum_{m=w+1}^{2 N} T_{w}[2 m-1]+4 T_{w}{ }^{2}(2 N-w)$ \\
\hline
\end{tabular}

TABLE II: A comparison of total number of operation between conventional FSD and FSD with power allocation.

\begin{tabular}{|c|c|c|}
\hline Case & Multiplications & Additions \\
\hline Conventional FSD, $T_{w}=16$ & 17250 & 44966 \\
\hline Conventional FSD, $T_{w}=32$ & 34274 & 143014 \\
\hline FSD with power allocation, $T_{w}=2$ & 3200 & 3566 \\
\hline
\end{tabular}

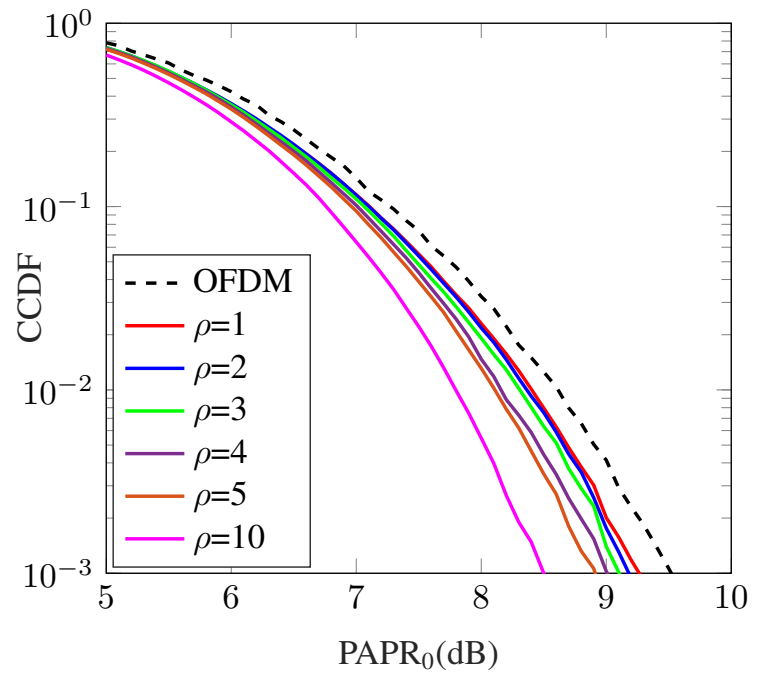

(a) $\alpha=4 / 5$

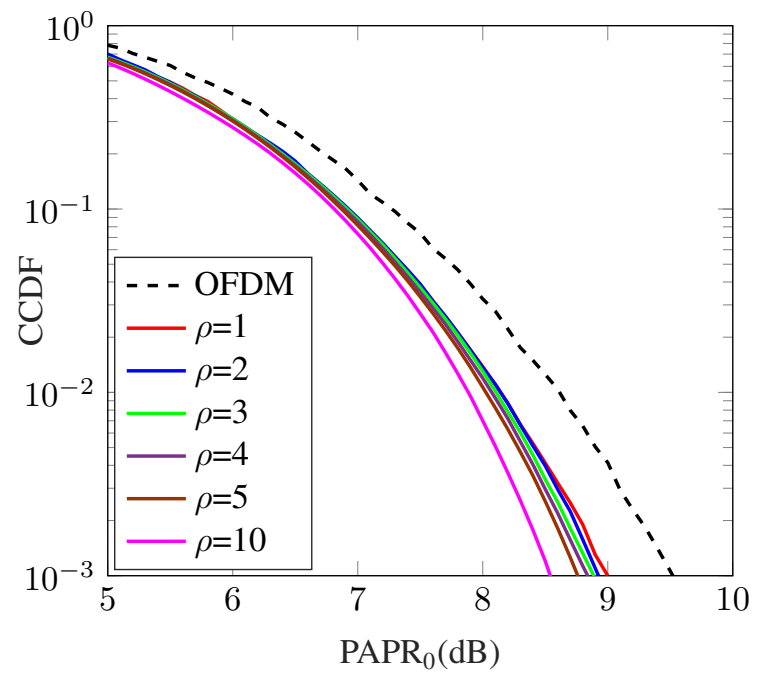

(b) $\alpha=2 / 3$

Fig. 6: PAPR distribution of SEFDM signals with different power ratios $(\rho$ ) and $N=16, M=4$, (a) $\alpha=4 / 5$, (b) $\alpha=2 / 3$.

conventional FSD with high number of subcarriers the correlation matrix is almost singular (refer to Fig. 2), thus, FSD is not capable to detect such a signal.

A numerical representation of the proposed method detector complexity, in terms of real-valued multiplications and real-valued additions, is summarised in Table I. A detailed computation of FSD complexity can be found in [18]. Table II calculates the complexity for the simulated BER results of Fig. 5(a) using Table. I. Clearly, the new method is orders of magnitude less complex than the stand-alone FSD detector without power allocation.

\section{PAPR Reduction}

In the previous work of [7], it was shown that the PAPR is dependent of the compression factor $(\alpha)$ and PAPR reduces by increasing the compression level, compared to OFDM. Thus, SEFDM signals are less prone to the non-linear effect when amplifiers are used, which is considered as an added advantage of SEFDM besides improving the spectral efficiency, specifically for satellite communication systems. Recently, [19] and [6] investigated the PAPR of multi-carrier faster than Nyquist (MFTN), which is similar to an SEFDM case with root raised cosine (RRC) shaped subcarriers [20]. The results agree with the previous conclusion of [7] that SEFDM or MFTN have a better PAPR behaviour than OFDM.

The PAPR performance is usually evaluated by the complementary cumulative distribution function (CCDF), which is defined as the probability that the PAPR surpasses a certain value $\left(\mathrm{PAPR}_{0}>0\right)$. Fig. 6 shows the PAPR distribution for two different compression values, given $N=16$ and $M=4$. From 
the figure, we can notice that the PAPR perfromance is improved by lowering $\alpha$ and/or increasing $\rho$. Thus, power allocation has the advantage of reducing the PAPR further compared to conventional SEFDM signals.

Methods introduced to reduce PAPR for OFDM, SEFDM and MFTN, such as selective windowing (SLW) [7], partial transmit sequence [19] and tone reservation PAPR reduction algorithm [21] can be applied here as well, for further PAPR reduction.

\section{CONCLUSIONS}

This work proposes a new power allocation method to SEFDM, where different subcarriers within the same symbol are allocated different power levels. A new system architecture based on double stage detection techniques has been designed, and the presented results prove that such power allocation is beneficial to SEFDM from different perspectives: It solves the main limitation of SEFDM signals, which relates to its ill-conditioning nature resulting from ICI; it reduces the detector complexity drastically compared to conventional SEFDM without power allocation, for the same error performance, with a particular case presented here for the suboptimal FSD detector; and finally the use of power allocation enhances the PAPR behaviour of SEFDM, which is essential for non-linear systems. Further investigation of this proposed method is the subject of ongoing work.

\section{ACKNOWLEDGMENT}

This work was supported in part by the Engineering and Physical Sciences Research Council (EPSRC), Impact Acceleration Account (IAA) Award and BT. We are also grateful to UCL for funding Hedaia Ghannam's $\mathrm{PhD}$ studies through the Overseas Research Student Award (ORS) and the UCL Dean of Engineering Sciences Award.

\section{REFERENCES}

[1] M. Rodrigues and I. Darwazeh, "A Spectrally Efficient Frequency Division Multiplexing Based Communications System," in Proc. 8th Int. OFDM Workshop, Hamburg, Nov. 2003, pp. $48-49$.

[2] D. Rainnie, Y. Feng, and J. Bajcsy, "On Capacity Merits of Spectrally Efficient fdm," in MILCOM 2015, Oct 2015, pp. 581586.

[3] I. Darwazeh, H. Ghannam, and T. Xu, "The First 15 Years of SEFDM: A Brief Survey," in 14th International Symposium on CSNDSP, July 2018

[4] T. Xu and I. Darwazeh, "Transmission Experiment of Bandwidth Compressed Carrier Aggregation in a Realistic Fading Channel," IEEE Trans. Veh. Technol., 2016.

[5] H. Ghannam and I. Darwazeh, "SEFDM Over Satellite Systems with Advanced Interference Cancellation," IET Communications, vol. 12, no. 1, pp. 59-66, 2018.

[6] S. Peng, A. Liu, L. Song, I. Memon, and H. Wang, "Spectral Efficiency Maximization for Deliberate Clipping-Based Multicarrier Faster-Than-Nyquist Signaling," IEEE Access, vol. 6, pp. 13617-13623, 2018.
[7] S. Isam and I. Darwazeh, "Peak to Average Power Ratio Reduction in Spectrally Efficient FDM Systems," in 2011 18th ICT, May 2011, pp. 363-368.

[8] T. Xu, S. Mikroulis, J. E. Mitchell, and I. Darwazeh, "Bandwidth Compressed Waveform for $60-\mathrm{GHz}$ Millimeter-Wave Radio over Fiber Experiment," J. of Lightwave Technol., vol. 34, no. 14, pp. 3458-3465, July 2016.

[9] Y. Wang, Y. Zhou, T. Gui, K. Zhong, X. Zhou, L. Wang, A. P. T. Lau, C. Lu, and N. Chi, "SEFDM Based Spectrum Compressed VLC System Using RLS Time-domain Channel estimation and ID-FSD Hybrid Decoder," in 42nd ECOC 2016, Sept 2016, pp. $1-3$.

[10] D. Nopchinda, T. Xu, R. Maher, B. C. Thomsen, and I. Darwazeh, "Dual Polarization Coherent Optical Spectrally Efficient Frequency Division Multiplexing," IEEE Photon. Technol. Lett, vol. 28, no. 1, pp. 83-86, Jan 2016.

[11] J. Zhou, Y. Qiao, Z. Yang, M. Guo, and X. Tang, "Capacity Limit for Faster-than-Nyquist Non-Orthogonal FrequencyDivision Multiplexing Signaling," in Scientific Reports, 2017.

[12] M. Jia, Z. Yin, Q. Guo, G. Liu, and X. Gu, "Downlink Design for Spectrum Efficient IoT Network," IEEE Internet Things J., vol. PP, no. 99, pp. 1-1, 2017.

[13] C. Y. Wong, R. S. Cheng, K. B. Lataief, and R. D. Murch, "Multiuser OFDM with Adaptive Subcarrier, Bit, and Power Allocation," IEEE J. Sel. Areas Commun., vol. 17, no. 10, pp. 1747-1758, 1999.

[14] L. Dai, B. Wang, Y. Yuan, S. Han, C. 1. I, and Z. Wang, "NonOrthogonal Multiple Access for 5G: Solutions, Challenges, Opportunities, and Future Research Trends," IEEE Commun. Mag., vol. 53, no. 9, pp. 74-81, September 2015.

[15] I. Kanaras, A. Chorti, M. R. D. Rodrigues, and I. Darwazeh, "Spectrally Efficient FDM Signals: Bandwidth Gain at the Expense of Receiver Complexity," in 2009 IEEE ICC, June 2009, pp. 1-6.

[16] L. G. Barbero and J. S. Thompson, "Fixing the Complexity of the Sphere Decoder for MIMO Detection," IEEE Trans. Wireless Commun., vol. 7, no. 6, pp. 2131-2142, June 2008.

[17] S. Isam and I. Darwazeh, "Characterizing The Intercarrier Interference of Non-Orthogonal Spectrally Efficient FDM System," in IEEE CSNDSP, 2012 8th International Symposium, July 2012, pp. 1-5.

[18] T. Xu, R. C. Grammenos, F. Marvasti, and I. Darwazeh, "An Improved Fixed Sphere Decoder Employing Soft Decision for the Detection of Non-Orthogonal Signals," IEEE Commun. Lett., vol. 17, no. 10, pp. 1964-1967, October 2013.

[19] S. Peng, A. Liu, K. Wang, and X. Liang, "PAPR Reduction of Multicarrier Faster-Than-Nyquist Signals with Partial Transmit Sequence," IEEE Access, vol. 5, pp. 24 931-24937, 2017.

[20] S. V. Zavjalov, S. V. Volvenko, and S. B. Makarov, "A Method for Increasing the Spectral and Energy Efficiency SEFDM Signals," IEEE Commun. Lett., vol. 20, no. 12, pp. 2382-2385, Dec 2016.

[21] E. O. Antonov, A. V. Rashich, D. K. Fadeev, and N. Tan, "Reduced Complexity Tone Reservation Peak-to-Average Power Ratio Reduction Algorithm for SEFDM Signals," in 2016 39th International Conference TSP, June 2016, pp. 445-448. 